\title{
The Interconnection between Malocclusion and Mouth Breathing
}

\author{
Clarissa S G da Fontoura*, Rodrigo Rocha Maia, Elisabeta Karl and Andrea Mantesso \\ Clinical assistant professor, University of Michigan, USA
}

Submission: August 24, 2021; Published: October 11, 2021

*Corresponding author: Clinical assistant professor, University of Michigan, 1011 N. University Avenue, Commons room 2133, Ann Arbor, MI 481091078, USA

\section{Abstract}

For decades, the impact of mouth breathing on malocclusion has been a contentious topic within dentists and otolaryngologist. When malocclusion is correlated with mouth breathing, patients display modifications in tooth and jaw positioning, facial height, and morphological changes in the oropharynx. However, the interaction between dentists and physicians is limited, even though it is in the best interest of the patients to be treated with a joint approach. This inadequate interaction is partially due to the lack of proper communication, limited interdisciplinary knowledge, and poor coordination between the professionals that should be involved in the case. For the patient, the interdisciplinary approach between dentists and otolaryngologists is beneficial, resulting in less costly and shorter treatments. The dentist, with an early diagnosis for a mouth breathing patient, ensures a correct treatment plan, potentially avoiding progression of the malocclusion as well as further dental and skeletal deformations. The otolaryngologist can improve the respiratory function and restore oral muscle balance. This will also prevent the establishment of a malocclusion, avoid the progression and/or prevent treatment relapses if a malocclusion is present. In this mini review we present how mouth breathing is associated with malocclusion, the most common facial phenotype in mouth breathers and discuss the dental management of malocclusion cases associated with mouth breathing.

\section{Introduction}

Abnormal oral habits have a significant role in the etiopathogenesis of malocclusions. Specifically, neuromuscular behaviors can develop to counteract dent alveolar malposition and inappropriate breathing activity [1]. Hence, the establishment of risk factors related to malocclusion, is critical to understand the impact of incorrect oral habits, such as mouth breathing, attributed with aberrant craniofacial growth. Malocclusion is characterized as a disruption of the correct relation between the maxilla and mandible as they come together in occlusion. Portrayed as the "disease of civilization", it has been a growing problem since the last century [2]. This condition is, in fact, the utmost prevalent craniofacial disorder impacting approximately $70 \%$ of American children [3]. The National Health Interview Survey has suggested that about 2.5 million children and 10 million young adults need orthodontic examination due to malocclusion. Furthermore, data from American Association of Orthodontists (AAO) indicates that over 3 million individuals must use fixed orthodontic appliances per year in United States (US) in order to correct occlusion disruption [3]. In the past years, malocclusion has been broadly researched to find mechanisms that contribute to craniofacial disorders. As a result, it was established that subjects typically develop malocclusion as a product of complex traits and interacting elements that affect craniofacial development during infancy and pubertal growth $[4,5]$. However, it is unknown how biological mechanisms, environmental and genetic factors affect the establishment of malocclusion.

Understanding how environmental and biological factors work in normal craniofacial development is crucial to understanding how they influence the establishment of malocclusion and mouth breathing. A normal craniofacial development relies on a synchronized interaction of the neurocranium and viscerocranium. According to Enlow, skeletal growth follows the mechanisms of bone remodeling, a balanced process of bone resorption and apposition, in which the spatial displacement is an outcome of pressures and/or tractions employed by neighboring tissues [6]. Moss's vision, recognized as the functional paradigm, launched in 1962, and later recognized as the principle of "form follows function", proposed that skeletal growth was a dynamic process in which craniofacial segments respond to growth as function demands. Further theories, such as Scott's and Moss's 
functional matrix theory, correlates craniofacial development with the downward and forward displacement of the facial structures $[7,8]$. However, malocclusion is not only caused by altered relationships between hard and soft components of the face and the unbalanced process of bone apposition and resorption during craniofacial growth [6].

Mouth breathing, an action that most commonly occurs during sleep is a condition often associated as a major risk factor for the onset of malocclusion [9-14]. There is, in fact, a feedback mechanism involved in the association of malocclusion and mouth breathing as the unbalanced oral function influences an abnormal craniofacial growth. In response, the abnormal relationship of the craniofacial structures also affects oral function [10]. Oral habits are more important during childhood for malocclusion development and their damaging effects have been described in several studies [9-15]. Every person who breathes through their mouth because of a pathological adaption, is referred to as a mouth breather. Mouth breathing is caused by a variety of factors, including anatomical obstructions such as, tonsil hypertrophy, septal deviation, nasal polyps, allergic rhinitis as well as deleterious oral habits [11,13,15-17]. The alteration of facial harmony and severity of deformation of the dental arches are dependent on the intensity, frequency, and duration of the conditions. Mouth breathing is particularly critical during development as it may negatively impact the craniofacial growth [18]. The aim of this review is to explore how malocclusion is developed as an indirect result of mouth breathing and how it can be managed.

\section{Craniofacial Abnormal Growth and Resulting}

\section{Phenotypes Associated with Mouth Breathing}

According to Moss's theory of functional matrix, proper nasal breathing warrants optimal craniofacial complex growth and development [8]. This hypothesis is based on the idea that a correct nasal respiratory activity positively interacts with the oral functions and head and neck structures leading to a well synchronized craniofacial development $[8,19]$. The incorrect function of the oral facial complex including the lips, mandiblemaxilla relationship, tongue, and/or oropharynx interferes with normal growth, development, or function of other oral structures because of a series of events or a lack of intervention at critical times, resulting in malocclusion and suboptimal oral health [1].

If habitual mouth breathing is present during the critical craniofacial growth period, an abnormal relationship of orofacial structures is established. As a result, deformations of craniofacial structures are observed including altered position of the head relative to the neck, anterior open bite, increased overjet, narrowing of the palatal plates and clockwise rotation of the mandible leading to an increase in facial height $[1,12,18]$. However, mouth breathing has not been found to be the sole or even the major cause of these conditions [18]. In this regard, previous literature has reported conflicting findings on the impact of mouth breathing on the maxilla and mandible, as well as the location of the maxilla relative to the skull base. Children with mouth breathing frequently present long adenoid faces. This phenotype is characterized by incompetent upper lip, a retro positioned hyoid bone, a narrow upper dental arch, retro positioned mandibular incisors, an increased anterior face height, a narrow or "V"-shaped maxillary arch, an increased mandibular plane angle, and a posteriorly rotated mandible [14]. These characteristics are, in fact, normally seen in individuals presenting class II malocclusion phenotype [20].

Although the studies have suggested mouth breathing as a risk factor for malocclusion, the etiology of this condition is still unclear. Knowledge inconsistencies lie in understanding the simultaneous influence of genetic factors in individuals with abnormal breathing activity. Previously it has been shown that malocclusion susceptibility is influenced by genetic factors [5]. Craniofacial phenotypes such as middle and lower facial proportions, arch dimensions and dental spacing have been found to have moderate to high heritability proportions $(>60 \%)$ as Class III malocclusion is suggested to have polygenic inheritance and autosomal dominance patterns, with incomplete penetrance [4].

On the other hand, studies of Class II malocclusion (commonly associated with mouth breathing) suggested a lower heritability pattern. For instance, associated phenotypes such as overjet and overbite show heritability varying from $28 \%$ to $53 \%$ respectively, suggesting a higher susceptibility to environmental factors $[21,22]$. Even though class II seems to have larger environmental factor influence compared to class III phenotypes, there is a bulk of evidence associating genetic variations and other specific phenotypes $[4,23-26]$. Therefore, the probability of an individual to develop malocclusion due to mouth breathing relies, in many cases, on the combination of factors happening during a specific period of time. To evaluate the risk level, it is important to analyze the timing of skeletal growth, genetic susceptibility and the presence or absence of mouth breathing activity of the patient.

\section{Management of Malocclusion Related Mouth Breathing}

Ongoing studies investigating the influence of abnormal breathing activities influencing craniofacial development have shown its clinical implications in the treatment and prevention of malocclusion [9-13]. The study of this relationship is fundamental for the treatment of malocclusion since the condition for a successful outcome is based on the elimination of the causes. Severe malocclusion will invariably end in craniofacial distortion, ultimately affecting oral function and esthetics as previously described [5]. The clinician's adoption of the optimal treatment regimen tailored to a precise diagnosis and understanding of the patient's growth potential, physiological and environmental factors determine the therapy's effectiveness. Treating class II malocclusion patients can be challenging due to relapses that are 
more frequent when the case increases in severity or is associated with risk factors such as mouth breathing.

Because the treatment starts at mixed dentition, the use of functional appliances is critical to inhibit the growth of the maxilla and enhance the growth of the mandible in a timely manner to decrease the severity of malocclusion [27]. Briefly, this treatment can be carried out in 2 steps using removable and fixed appliances according to the growth period of the patient. In the mixed dentition, the class II malocclusion triggered by mouth breathing is managed with an early functional corrector (with the use of myofunctional appliances), orthodontic treatment such as rapid maxillary expansion, as well as combined orthodontic and orthognathic treatment. The treatment of Class II malocclusion when in permanent dentition, is usually done with fixed orthodontic appliances for correcting phenotypes such as midline misalignment, open bite, overjet, posterior cross bite, correct mandibular vertical growth as well as teeth crowding and rotations $[27,28]$.

To ensure a proper orthodontic diagnosis and treatment of malocclusion, it is critical to understand basic principles of craniofacial development. When health care providers develop a treatment plan for malocclusion triggered by mouth breathing or other orofacial dysfunctional habits, they invariably must acknowledge the critical role of interdisciplinary approaches. Many orthodontic treatments begin during childhood, around the age of seven [1]. However, signs and symptoms of mouth breathing can appear much earlier. In this scenario, if a coordinated approach is not established, two situations can occur:

i. Mouth breathing is left untreated, and a malocclusion may develop as consequence.

ii. Orthodontic treatment is performed without considering mouth breathing, invariably leading to an increased risk of orthodontic relapse.

Therefore, routine evaluations for abnormal breathing activities and subsequent referral to the specialized professionals should be a requirement to ensure a proper treatment plan.

To summarize, the otolaryngologist should keep in mind that mouth breathers have a high risk of developing malocclusions and should refer patients with this condition to dentists, especially during infancy and pubertal growth. Likewise, the dentist, when treating a case of malocclusion associated with mouth breathing should refer the patient to an otolaryngologist for an interdisciplinary approach that will result in more effective treatment and less probability of recurrence.

\section{Discussion}

Fordecades, the impact of mouth breathing on malocclusion has been a contentious topic between orthodontic and otolaryngology professionals. While a growing number of researchers confirm the association between mouth breathing and craniofacial development [11,13,15-17], preceding ones did not believe that breathing abnormalities play a key role in the craniofacial development process $[29,30]$. This perception is questionable, in part, due to the subjective nature of the criteria's used to diagnose mouth breathing. On the other hand, while informative, many malocclusion studies are limited by modest sample sizes, unclear generalized information and, most importantly, by the use of exclusive categorical phenotypes (i.e. class II malocclusion) which do not capture the phenotypic complexity of the condition. As mentioned before, genetic factors play an important role in influencing the constitution of the craniofacial phenotypic variations. Therefore, a critical obstacle for studying malocclusion conditions lies in the complex nature and the ability to capture the full spectrum of malocclusion phenotypes.

These limitations can be mitigated using more sophisticated methods for the analysis of shape to minimize the impact of the phenotypic variability and detailed descriptions of the environmental factors present. The study of variables associated with malocclusions etiology is also critical for developing public health strategies targeted at preventing and therapeutically intercepting this health condition. Around 20\% of the US population present abnormalities of occlusion. Class II malocclusion it is estimated to affect $15 \%$ of the population while Class III malocclusion affects just 1\% [3]. Thus, growth modification appliances can be used alone or in combination with fixed orthodontic therapy to address malocclusions. Yet, severe cases of malocclusion sometimes require orthognathic and dental surgery.

As a result, the estimated Medicaid expenditure on orthodontic treatments in the United States ranges from \$29.5 million to $\$ 75.2$ million per year -FY 2013, 2014 [31]. This expenditure is obviously higher if moderate and mild cases of malocclusions are included. The interdisciplinary referral between dentists and otolaryngologists, particularly for patients in growth ages, allow for less severe cases of the malocclusion potentially resulting in shorter and cheaper treatments. The complex relation between malocclusion and mouth breathing highlights the importance of further research to ensure an early diagnosis, preventive measures and proper multi professional treatments. Future research should also look at identifying functional variants predisposed to abnormal breathing activity correlated to malocclusion development.

\section{Conclusion}

In conclusion, better diagnostic tools and interdisciplinary approaches that avoid costly and time-consuming treatments are necessary. Therefore, it is critical to recognize causal factors and phenotypic characteristics associated with cases of malocclusion related to mouth breathing. The correct treatment for these cases should involve dentists and otolaryngologists, aimed not only at 
correcting the dental and jaw positioning, but also the oral and respiratory function.

\section{References}

1. D'Onofrio L (2019) Oral dysfunction as a cause of malocclusion. Orthod Craniofac Res 22(1): 43-48.

2. Warren JJ, Bishara SE, Yonezu T (2003) Tooth size-arch length relationships in the deciduous dentition: A comparison between contemporary and historical samples. Am J Orthod Dentofacial Orthop 123(6): 614-619.

3. Proffit WR, Fields HW Jr, Moray LJ (1998) Prevalence of malocclusion and orthodontic treatment need in the united states: Estimates from the nhanes iii survey. Int J Adult Orthodon Orthognath Surg 13(2): $97-$ 106.

4. Moreno Uribe LM, Miller SF (2015) Genetics of the dentofacial variation in human malocclusion. Orthod Craniofac Res 18(1): 91-99.

5. Joshi N, Hamdan AM, Fakhouri WD (2014) skeletal malocclusion: A developmental disorder with a life-long morbidity. J Clin Med Res 6(6): 399-408.

6. Enlow DH, Robert E Moyers (1982) Handbook of facial growth. $\left(2^{\text {nd }}\right.$ Eds) Wb saunders company.

7. Moss ML (1954) Growth of the calvaria in the rat; the determination of osseous morphology. Am J Anat 94(3): 333-361.

8. Moss ML, Salentijn L (1969) The primary role of functional matrices in facial growth. Am J Orthod 55(6): 566-577.

9. Fields HW, Warren DW, Black K, Phillips CL (1991) Relationship between vertical dentofacial morphology and respiration in adolescents. Am J Orthod Dentofacial Orthop 99(2): 147-154.

10. Hultcrantz E, Löfstrand Tideström B (2009) The development of sleep disordered breathing from 4 to 12 years and dental arch morphology. Int J Pediatr Otorhinolaryngol 73(9): 1234-1241.

11. Mattar SE, Valera FC, Faria G, Matsumoto MA, Anselmo Lima WT (2011) Changes in facial morphology after adenotonsillectomy in mouth-breathing children. Int J Paediatr Dent 21(5): 389-396.

12. Harari D, Redlich M, Miri S, Hamud T, Gross M (2010) The effect of mouth breathing versus nasal breathing on dentofacial and craniofacial development in orthodontic patients. Laryngoscope 120(10): 20892093.

13. Chung Leng Muñoz I, Beltri Orta P (2014) Comparison of cephalometric patterns in mouth breathing and nose breathing children. Int J Pediatr Otorhinolaryngol 78(7): 1167-1172.

14. Wysocki J, Krasny M, Skarzyński PH (2009) Patency of nasopharynx and a cephalometric image in the children with orthodontic problems. Int J Pediatr Otorhinolaryngol 73(12): 1803-1809.

15. Lessa FC, Enoki C, Feres MF, Valera FC, Lima WT, et al. (2005) Breathing mode influence in craniofacial development. Braz J Otorhinolaryngol 71(2): 156-160.

16. Rossi RC, Rossi NJ, Yamashita HK, Pignatari SS (2015) Dentofacial characteristics of oral breathers in different ages: A retrospective casecontrol study. Prog Orthod 16: 23.
17. Chambi Rocha A, Cabrera Domínguez ME, Domínguez Reyes A (2018) Breathing mode influence on craniofacial development and head posture. J Pediatr 94(2): 123-130.

18. Management of the developing dentition and occlusion in pediatric dentistry (2017) Pediatr Dent 39(6): 334-347.

19. Cooper BC (1989) Nasorespiratory function and orofacial development. Otolaryngol Clin North Am 22(2): 413-441.

20. Fraga WS, Seixas VM, Santos JC, Paranhos LR, César CP (2018) Mouth breathing in children and its impact in dental malocclusion: A systematic review of observational studies. Minerva Stomatol 67(3): 129-138.

21. Naini FB, Moss JP (2004) Three-dimensional assessment of the relative contribution of genetics and environment to various facial parameters with the twin method. Am J Orthod Dentofacial Orthop 126(6): 655665.

22. Townsend G, Hughes T, Luciano M, Bockmann M, Brook A (2009) Genetic and environmental influences on human dental variation: A critical evaluation of studies involving twins. Arch Oral Biol 54(1): S4551.

23. Moreno Uribe LM, Vela KC, Kummet C, Dawson DV, Southard TE (2013) Phenotypic diversity in white adults with moderate to severe class iii malocclusion. Am J Orthod Dentofacial Orthop 144(1): 32-42.

24. Weaver CA, Miller SF, da Fontoura CS, Wehby GL, Amendt BA, et al. (2017) Candidate gene analyses of 3-dimensional dentoalveolar phenotypes in subjects with malocclusion. Am J Orthod Dentofacial Orthop 151(3): 539-558.

25. Sun Z, da Fontoura CSG, Moreno M, Holton NE, Sweat M, et al. (2018) Foxo6 regulates hippo signaling and growth of the craniofacial complex. PLoS Genet 14(10): e1007675.

26. da Fontoura CS, Miller SF, Wehby GL, Amendt BA, Holton NE, et al. (2015) Candidate gene analyses of skeletal variation in malocclusion. ] Dent Res 94(7): 913-920.

27. Lyu L, Zhao Z, Tang Q, Zhao J, Huang H (2021) Skeletal class ii malocclusion caused by mouth breathing in a pediatric patient undergoing treatment by interceptive guidance of occlusion. J Int Med Res 49(6): 3000605211021037.

28. Li X, Wang H, Li S, Bai Y (2019) Treatment of a class ii division 1 malocclusion with the combination of a myofunctional trainer and fixed appliances. Am J Orthod Dentofacial Orthop 156(4): 545-554.

29. Tourné LP (1991) Growth of the pharynx and its physiologic implications. Am J Orthod Dentofacial Orthop 99(2): 129-139.

30. Tourne LP, Schweiger J (1996) Immediate postural responses to total nasal obstruction. Am J Orthod Dentofacial Orthop 110(6): 606-611.

31. Minick G, Tilliss T, Shellhart WC, Newman SM, Carey CM, et al. (2017) Comparison of orthodontic medicaid funding in the united states 2006 to 2015. Front Public Health 5: 221. 
(C) Commons Attribution 4.0 License

(CC) DY 10.19080/GJO.2021.24.556147

\section{Your next submission with Juniper Publishers will reach you the below assets}

- Quality Editorial service

- Swift Peer Review

- Reprints availability

- E-prints Service

- Manuscript Podcast for convenient understanding

- Global attainment for your research

- Manuscript accessibility in different formats ( Pdf, E-pub, Full Text, Audio)

- Unceasing customer service

Track the below URL for one-step submission https://juniperpublishers.com/online-submission.php 